\title{
ESTUDIO FÍSICO-QUÍMICO DE LA CARNE DE LA RAZA BOVINA MARISMEÑA EN DIFERENTESSISTEMAS DE TERMINACIÓN
}

\author{
PHYSIC-CHEMICALSTUDY OF THE MARISMEÑA CATTLE BREED MEAT \\ IN DIFFERENT FATTENING SYSTEMS
}

\author{
Nogales, S. ${ }^{*}$, Bressan, M.C. ${ }^{2}$, Vaz, A.P. ${ }^{2}$, Delgado, J.V. ${ }^{1}$ y Camacho, M.E. ${ }^{3}$ \\ 'Departamento de Genética. Universidad de Córdoba. Córdoba. España. ”seio21@hotmail.com \\ ${ }^{2}$ Instituto Nacional dos Recursos Biológicos. Unidade de Genética, Reprodução e Melhoramento Animal. \\ INRB, IP. Fonte Boa. Vale de Santarém. Portugal. \\ ${ }^{3}$ IFAPA. Alameda del Obispo. Consejería de Innovación, Ciencia y Empresa. Córdoba. España.
}

\section{Palabras clave adicionales \\ Caracterización productiva. Conservación.}

\section{RESUMEN}

El bovino Marismeño es una entidad racial asilvestrada incluida en un sistema agroecológico prezootécnico, el Espacio Natural de Doñana que no encuentra una vía de comercialización adecuada que la diferencie de lo típicamente comercial, y, por tanto, agregue valor para competir mediante una calidad diferenciada. En este trabajo se presentan los resultados preliminares del estudio físico-químico de la carne. Se han estudiado 17 animales de la raza en dos sistemas de terminación diferentes, su entorno natural (extensivo) y un cebadero comercial (intensivo). La carne mostró diferencias significativas entre los sistemas de producción para los resultados de los análisis químicos y el color a las 24 horas postmortem. Los animales del sistema extensivo mostraron carnes magras, con un alto porcentaje de humedad, y una coloración con menores intensidades de rojo $(p<0,01)$ y amarillo $(p<0,001)$, comparada con los animales del intensivo. Los resultados demuestran una diferenciación entre ambos sistemas, a la vez que se hallan algunas características que conducen a la diferenciación del producto de esta raza bovina en el mercado.

\section{SUMMARY}

Marismeña cattle breed is a feral population included in a pre-zootechnic agro-ecosystem, the

$\overline{\text { Presentado al }}$ Congreso SERGA (2010, Asturias).

\author{
Additional KEYWORDS \\ Productive characterization. Conservation.
}

Doñana protected area. In spite of these characteristics so exclusives, the absence of characterization of their products difficults the finding of a way of commercialization differentiated of the typical commerce, adding value to product by way of a differentiated quality. The preliminary results of a meat physic-chemical study are presented. Seventeen animals of the breed have been studied reared in two different systems of fattening: its natural context and a commercial fattening station. Meat shown significant differences between the management systems for the results of the chemical analysis and colour at 24 hours posmortem. Animals belonging to the extensive system shown meat low in fat, with a high percentage of humidity, and colour with lower tone of red $(p<0.01)$ and yellow $(p<0.001)$, when compared with intensive animals. Results demonstrated a differentiation between both systems, together with some arguments to differentiate the products of this breed in the market.

\section{INTRODUCCIÓN}

La vaca Marismeña habita en el Parque Nacional de Doñana de forma asilvestrada. Su alimentación se basa exclusivamente en lo que el medio proporciona, y el manejo se reduce a las épocas de recogida, sanea- 
miento, reposición y venta del ganado. $\mathrm{Su}$ situación de peligro de extinción ha hecho que se pongan en marcha una serie de mecanismos para conservarla. Uno de los problemas es la comercialización de estos animales, debido a que sus características cárnicas no se adaptan a los parámetros comerciales generales, restándoles valor. Teniendo en cuenta las características de la raza y su particular sistema de producción la caracterización de la carne puede facilitar su comercialización como producto diferenciado por canales diferentes a los habituales.

\section{MATERIAL Y MÉTODOS}

Se identificaron 20 animales, 10 machos y 10 hembras, nacidos en la misma paridera. Los primeros 6 meses de edad fueron criados en libertad en el Parque Nacional de Doñana. Posteriormente, 5 machos y 5 hembras, se trasladaron a un cebadero comercial de terneros en la provincia de Córdoba, grupo denominado como sistema intensivo (I). La alimentación se llevó a cabo con un pienso cuya composición se refleja en la tabla I. Los 10 restantes prosiguieron en su sistema natural hasta el sacrificio, grupo denominado como sistema extensivo (E). Los animales se sacrificaron en el mismo momento, con una edad aproximada de 18 meses, en el matadero comercial con homologación europea de la empresa COVAP (Cooperativa del Valle de los Pedroches). Del grupo E se produjo la pérdida de 3 machos, los cuales no pudieron ser sacrificados. El sacrificio fue realizado según la reglamentación de la UE (Reglamento 1099/ 2009). Las canales se mantuvieron en cámara de oreo durante 24 horas a $2^{\circ} \mathrm{C}$. A continuación se realizó el despiece de la semicanal izquierda y se tomaron muestras del $M$. longissimus thoracis para el análisis centesimal. El pH fue medido en este músculo al sacrificio y a las 24 horas postmortem con pHmetro portátil pH25 (Crison Instruments, S.A., Barcelona, España). El color se tomó con un espectrofotómetro
Tabla I. Composición porcentual del pienso, por ingredientes, consumido por los animales del sistema intensivo o cebadero. (Percentage composition of the animal foods, consumed by the individuals in the fattening station).

\begin{tabular}{lc}
\hline Ingredientes & Porcentajes \\
\hline Maíz & 39,93 \\
Trigo & 24,84 \\
Cebada & 22,18 \\
Soja & 10,65 \\
C. minerales & 1,33 \\
Sal & 1,06 \\
\hline
\end{tabular}

CM-700d (Konica Minolta Holdings, Inc., Tokio, Japón), con iluminante D65 y observador de $10^{\circ}$, obteniéndose los parámetros $L^{*}, a^{*}$ y b* de acuerdo con el sistema CIE (CIE, 1976). En el análisis centesimal, siguiendo el protocolo de la AOAC (2005), se obtuvo la composición de la carne en proteína bruta por el método micro Kjedahl, la grasa por el método Soxhlet, la humedad en estufa, y las cenizas en horno mufla. El análisis estadístico de los datos se realizó por el procedimiento GLM del paquete estadístico SAS (2004).

\section{RESULTADOSYDISCUSIÓN}

Al sacrificio, los animales presentaron diferencias significativas $(\mathrm{p}<0,001)$ en los pesos entre sistemas, $282,57 \pm 42,04$ para extensivo, y 428,8 $\pm 65,96$ para intensivo. En la tabla II se presentan los datos referentes

Tabla II. Medias \pm desviaciones estándar y nivel de significación de las medidas del pH al sacrificio y a las 24 horas. (Means \pm standard deviations, and level of signification of the means in $\mathrm{pH}$ at slaughtering, and at 24 hours).

\begin{tabular}{llll}
\hline & \multicolumn{2}{c}{$\begin{array}{c}\text { Sistema de terminación } \\
\text { Extensivo }\end{array}$} & Intensivo \\
& & \\
\hline $\mathrm{pH}$ sacrificio & $6,23 \pm 0,09$ & $6,06 \pm 0,26$ & $\mathrm{~ns}$ \\
$\mathrm{pH} 24$ horas & $5,69 \pm 0,12$ & $5,47 \pm 0,02$ & $* * *$ \\
\hline
\end{tabular}




\section{ANÁLISIS FÍSICO-QUÍMICO DE CARNE DE LA RAZA MARISMEÑA}

al $\mathrm{pH}$. Se puede observar que no se hallaron diferencias al sacrificio. A las 24 horas postmortem las diferencias entre los sistemas de terminación son notables $(\mathrm{p}<0,001)$. Los animales del sistema I muestran un descenso mayor del $\mathrm{pH}$ sugiriendo reservas de glucógeno más altas como consecuencia de haber recibido una dieta con un elevado aporte energético (Neath et al., 2007). A esto ha de sumarse que los animales del sistema de terminación extensivo posiblemente sufran un mayor estrés, con el consecuente gasto energético en las fases previas al sacrificio, debido a la ausencia de manejo durante su crecimiento (Muir et al., 1998). Sin embargo, los animales de ambos sistemas de terminación muestran valores de $\mathrm{pH}$ dentro del intervalo considerado adecuado para la comercialización de la carne (Mach et al., 2008). En la tabla III figuran los resultados relativos al color de la carne. Al sacrificio, los animales del sistema E mostraron una carne más oscura. Esta diferencia no fue hallada a las 24 horas, siendo las medias del parámetro $\mathrm{L}^{*}$ semejantes $(p>0,05)$. A pesar de que la grasa intramuscular que presentaron los animales del sistema I fue superior en más de 4 veces, no se denotan diferencias en la luminosidad, lo

Tabla III. Medias, desviaciones \pm estándar y nivel de significación para los parámetros del color medidos al sacrificio y a las 24 horas. (Means \pm standard deviations, and level of signification of the means of colours measured at slaughtering, and at 24 hours).

\begin{tabular}{lccc}
\hline & \multicolumn{2}{c}{$\begin{array}{c}\text { Sistema de terminación } \\
\text { Extensivo }\end{array}$} & Intensivo \\
& & \\
\hline $\mathrm{L}^{*}$ sacrificio & $18,43 \pm 2,05$ & $28,40 \pm 3,67$ & $* * *$ \\
$\mathrm{~L}^{*} 24$ horas & $29,99 \pm 1,05$ & $32,71 \pm 8,82$ & $\mathrm{~ns}$ \\
$\mathrm{a}^{*}$ sacrificio & $8,80 \pm 0,87$ & $6,25 \pm 1,79$ & $* *$ \\
$\mathrm{a}^{*} 24$ horas & $7,76 \pm 0,83$ & $11,11 \pm 2,09$ & $* *$ \\
$\mathrm{~b}^{*}$ sacrificio & $7,91 \pm 1,68$ & $6,20 \pm 0,64$ & $*$ \\
$\mathrm{~b}^{*} 24$ horas & $5,38 \pm 2,63$ & $11,25 \pm 1,31$ & $* *$ \\
\end{tabular}

${ }^{*} p<0,05 ;{ }^{* *} p<0,01 ;{ }^{* * *} p<0,001 ;$ ns: No significativo. que se cree debido a que la grasa que los animales de raza Marismeña depositaron en músculo se encontró formando bolas o regiones aparentemente circulares que fueron evitadas en las mediciones. Por otro lado, el mayor $\mathrm{pH}$ en los animales de E coincide con los resultados de $\mathrm{L}^{*}$ cuya media es ligeramente inferior, dando una carne levemente más oscura (Priolo et al., 2001). Al estudiar el índice de rojo (a*) se aprecia que la carne de I obtuvo valores mayores a las 24 horas. Es posible que los animales de este sistema muestren una capacidad mayor de oxigenación de los pigmentos del color, presentando formas de las hemoproteínas que tienen una tonalidad roja brillante (Mancini et al., 2005). Por otra parte, el índice de amarillo ( $\left.b^{*}\right)$ fue superior en los animales de I a las 24 horas, lo que no coincide con Kerth et al. (2007), que indican que los animales de pastos presentan en la carne gran cantidad de $\beta$-carotenos, mostrando carnes más amarillas. Esto sugiere que los pastos donde se alimentan los animales de la raza Marismeña tienen en su composición un bajo porcentaje de este pigmento. Los componentes del análisis químico de la carne (tabla IV) fueron afectados por el sistema de terminación, con excepción del porcentaje de cenizas $(p>0,05)$. La humedad se incremento en los animales del sistema E en un $4,13 \%$ ( $p<0,001$ ), mientras que la composición en proteínas fue levemente superior en los de I. La variación en los porcentajes de grasa intramuscular entre ambos sistemas se determinó en un $2,96 \%$ (con mayor contenido de grasa en animales del sistema I), diferencia que posiblemente resultó del mayor aporte energético en la dieta (Realini et al., 2004), y las diferencias en el desarrollo, que se constatan observando la variación en los pesos de sacrificio. El porcentaje hallado en los animales de I es de los mayores encontrados en la bibliografía entre otras razas autóctonas españolas. Por el contrario, el $0,95 \%$ de grasa intramuscular que se halló en la carne 
Tabla IV. Medias \pm desviaciones estándar y nivel de significación de las variables medidas en la composición centesimal de la car$n e$. (Means \pm standard deviations, and level of signification of the variables measured in the meat composition).

\begin{tabular}{lccc}
\hline & \multicolumn{2}{c}{ Sistema de terminación } & \\
& Extensivo & Intensivo & \\
\hline Humedad & $76,11 \pm 0,88$ & $71,98 \pm 1,83$ & $* * *$ \\
Proteínas & $21,96 \pm 0,76$ & $22,95 \pm 0,83$ & $*$ \\
Grasa & $0,95 \pm 0,43$ & $3,91 \pm 1,58$ & $* * *$ \\
Cenizas & $0.98 \pm 0,03$ & $0.96 \pm 0,03$ & ns \\
\hline
\end{tabular}

${ }^{*} p<0,05 ;{ }^{* *} p<0,001 ;$ ns: No significativo.

de los animales criados en los pastos de Doñana es de los más bajos (Cañeque y

\section{BIBLIOGRAFÍA}

AOAC. 2005. Official Methods of Analysis of the Association of Official Analytical Chemists. $18^{\text {th }}$ ed. Arlington.

Cañeque, V. y Sañudo, C. 2000. Metodología para el estudio de la calidad de la canal y de la carne en rumiantes. Instituto Nacional de Investigación y Tecnología Agraria y Alimentaria. Ministerio de Ciencia y Tecnología. Madrid. España. $255 \mathrm{pp}$.

CIE. 1976. Recommendations on uniform color spaces-color difference equations. Psycometric color terms. Suplement № 2. Commission Internationale de l'Éclaraige. París.

Kerth, C.R., Braden, K.W., Cox, R., Kerth, L.K. and Rankins, Jr., D.L. 2007. Carcass, sensory, fat color, and consumer acceptance characteristics of Angus-cross steers finished on ryegrass (Lolium multiflorum) forage or on a highconcentrate diet. Meat Sci., 75: 324-331.

Mach, N., Bach, A. Velarde, A. and Devant, M. 2008. Association between animal, transportation, slaughterhouse practices, and meat $\mathrm{pH}$ in beef. Meat Sci., 78: 232-238.

Mancini, R.A. and Hunt, M.C. 2005. Review: Current research in meat color. Meat Sci., 71: 100-121.

Muir, P.D., Beaker, J.M. and Brown, M.D. 1998.
Sañudo, 2000).

En conclusión, los resultados, preliminares con pequeña muestra y sin haber considerado el sexo, podrían indicar diferencias entre la carne de la raza Marismeña y de otras razas próximas, influido por su sistema de producción característico.

\section{AGRADECIMIENTOS}

A Raúl Sanz por el apoyo que ha brindado COVAP a través de su persona. A la Asociación Nacional de Criadores de Ganado Marismeño por el apoyo a este trabajo, desde los fondos otorgados por la Consejería de Agricultura de la Junta de Andalucía. Y a la Estación Biológica de Doñana por su labor en la conservación de la raza, y la cesión de los animales.

Effects of forage- and grain-based feeding systems on beef quality: A review. New Zeal. J. Agri. Res., 41: 623-635.

Neath, K.E., Del Barrio, A.N., Lapitan, R.M., Herrera, J.R.V., Cruz, L.C., Fujihara, T., Muroya, S., Chikuni, K., Hirabayashi, M. and Kanai, Y. 2007. Difference in tenderness and $\mathrm{pH}$ decline between water buffalo meat and beef during postmortem aging. Meat Sci., 75: 499-505.

Priolo, A., Micol, D. and Agabriel, J. 2001. Effects of grass feeding systems on ruminant meat colour and flavour: A review. Anim. Res., 50: 185-200.

Realini, C.E., Duckett, S.K., Brito, G.W., Dalla Rizza, M. and De Mattos, D. 2004. Effect of pasture vs. concentrate feeding with or without antioxidants on carcass characteristics, fatty acid composition, and quality of Uruguayan beef. Meat Sci., 66: 567-577.

SAS. Statistical Analyses Systems. 2004. User's Guide: Statistic. SAS Institute. Cary. NC. 176 pp. UE. 2009. Reglamento CE № 1099/2009 del Consejo de 24 de septiembre de 2009, relativo a la protección de los animales en el momento de la matanza. Diario Oficial de la Unión Europea. Bruselas. Bélgica. 